This is the post peer-review accepted manuscript of:

Zabini, F., Calisti, A., Dardari, D., \& Conti, A. (2016). Random Sampling via Sensor Networks: Estimation Accuracy vs. Energy Consumption. In 2016 24th European Signal Processing Conference (eusipco) (pp. 130-134). New York: leee. https://doi.org/10.1109/EUSIPCO.2016.7760224

The published version is available online at: . https://doi.org/10.1109/EUSIPCO.2016.7760224

C 2016 IEEE. Personal use of this material is permitted. Permission from IEEE must be obtained for all other uses, in any current or future media, including reprinting/republishing this material for advertising or promotional purposes, creating new collective works, for resale or redistribution to servers or lists, or reuse of any copyrighted component of this work in other works 


\title{
Random Sampling via Sensor Networks: Estimation Accuracy vs. Energy Consumption
}

\author{
Flavio Zabini, Alex Calisti, Davide Dardari, and Andrea Conti
}

\begin{abstract}
The estimation of spatial processes from sparse sensing nodes is fundamental for many applications, including environmental monitoring and crowd-sourcing. In this paper, we analyze the impact of measurement errors on the estimation of a finite-energy signal sampled by a set of sensors randomly deployed in a finite $d$-dimensional space according to homogeneous Poisson Point Process. The optimal linear space invariant interpolator is derived. Based on such an interpolator, analytical expressions of both the estimated signal energy spectral density and the normalized estimation mean square error are obtained. An asymptotic analysis for high sensors density with respect to the signal bandwidth is given for scenarios subjected to estimation energy constraint. The normalized estimation mean square error is derived for large wireless sensor networks with constraints on the capacity-per-volume and on battery duration.
\end{abstract}

\section{INTRODUCTION}

Self-organizing wireless sensor networks (WSN) has attracted considerable attention in the last years [1]. The possibility to create a network infrastructure composed of lowcost, small-size and energy-limited sensing devices (sensors) has created a large number of applications in smart home, environmental monitoring, crowdsensing and Internet of Things.

For the classical regular sampling, the well-known Whittaker-Kotelnikov-Shannon sampling theorem states that a signal can be perfectly reconstructed from its samples provided that the sampling frequency is larger than twice its bandwidth. For the irregular sampling, a theorem of Landau [2], [3] establishes necessary conditions on samples density for the perfect estimation. In the case of a random sampling in time, the estimation accuracy is usually evaluated in the terms of mean square error (MSE) [4]. In particular, if the sample positions are the output of a stationary Poisson point process (PPP), Marvasti [5] shows that the reconstruction is still possible via ideal low pass (ILP) filtering provided that the average samples density is higher than twice the signal bandwidth, and that the spectrum of the estimated signal is that of the original signal immersed in a white noise floor.

Recently, the extension of Marvasti's result to a multidimensional domain has gained interest due to its application on WSN for environmental monitoring [6]-[10]. The assumption that nodes in a WSN are deployed according to a homogeneous PPP (homogeneous in $\mathbb{R}^{d}$ corresponds to stationary in $\mathbb{R}$ ) is widely adopted [11]-[14]. ${ }^{1}$ However, many works do not

This work has been performed in part within the EU H2020 Project XCycle (grant no. 635975).

F. Zabini, A. Calisti, D. Dardari are with DEI, University of Bologna. Email: \{flavio.zabini2, alex.calisti, davide.dardari\}@unibo.it

A. Conti is with ENDIF, University of Ferrara. E-mail: a.conti@ieee.org

${ }^{1}$ Multidimensional signal reconstruction from inhomogeneous Poisson sampling has been analyzed in [15], where the optimal linear space invariant (LSI) interpolator is derived accounting for random sampling and signal properties. consider that the ILP filtering is no more optimal for irregular sampling, as widely known in the one-dimension domain in case of jitter [16]-[18]. Moreover, in a realistic sensing scenario, the distortion due to measurement errors and sensors energy consumption have to be considered [19]-[21].

In this paper, by following the approach in [15], we derive the optimal LSI interpolator minimizing the normalized estimation mean square error (NEMSE) by jointly taking into account random sampling, measurement errors and energy consumption. In particular, the estimated signal energy spectral density (ESD) and NEMSE are derived as functions of important parameters such as samples density and distortion. These results aim to answer the following question in multidimensional signal estimation: given a certain amount of energy spent for the estimation, is it better to have few accurate samples or many inaccurate ones? Anyway, in an actual WSN, the most relevant estimation energy constraint is not on the whole network, but on each sensor, due to the battery duration limitation. In addition, a constraint on the capacity of each sensor for sending the samples to the interpolation entity has to be taken into account. Therefore, another relevant question is: when the estimation accuracy of a large WSN is dominated by the capacity-per-volume and when by the sensor lifetime? By modeling the communication channel between each nodes and the interpolator as an erasure channel, and by considering the capacity-per-volume as a constraint for a large WSN, we derive a simple analytical expression for the NEMSE as a function of both the estimation rate and the capacity-per-volume.

\section{SySTEM MODEL}

Consider the signal $z(\mathbf{x}) \in \mathbb{C}$ with support $\mathcal{A} \subseteq \mathbb{R}^{d}$, finite energy $E_{z}$, and ESD $\mathcal{E}_{z}(\boldsymbol{\nu})$, where $\mathbf{x}, \boldsymbol{\nu} \in \mathbb{R}^{d}$. Let $\mathcal{F}\{\cdot\}$ and $\delta(\cdot)$ be the Fourier transformation and the Dirac delta generalized function in $\mathbb{R}^{d}$, respectively [22]. The homogeneous Poisson sampling process can be expressed as

$$
\mathcal{P}(\mathbf{x})=\sum_{n \in \mathcal{N}(\boldsymbol{\Pi})} \delta\left(\mathbf{x}-\mathbf{x}_{n}\right)
$$

where $\boldsymbol{\Pi}$ is the homogeneous PPP in $\mathbb{R}^{d}$ with intensity $\rho$ [23], $\mathbf{x}_{n}$ denotes the position of the $n$-th sample according to $\boldsymbol{\Pi}$, and $\mathcal{N}(\cdot)$ is the index set operator. It is known [24] that

$$
\begin{aligned}
\mathbb{E}\{\mathcal{P}(\mathbf{x})\} & =\rho \\
\mathbb{E}\{\mathcal{P}(\mathbf{x}) \mathcal{P}(\mathbf{x}-\boldsymbol{\tau})\} & =\rho^{2}+\rho \delta(\boldsymbol{\tau}) .
\end{aligned}
$$

The sampled signal with measurement errors is given by

$$
\mathbf{z}_{\epsilon}(\mathbf{x})=\sum_{n \in \mathcal{N}\left(\boldsymbol{\Pi}_{\mathcal{A}}\right)} \hat{\mathbf{z}}_{n} \delta\left(\mathbf{x}-\mathbf{x}_{n}\right)
$$


where $\mathcal{N}\left(\boldsymbol{\Pi}_{\mathcal{A}}\right)$ is the index set of the points of $\boldsymbol{\Pi}$ falling in $\mathcal{A}$. The $n$-th sample affected by measurement is

$$
\hat{\mathbf{z}}_{n}=z\left(\mathbf{x}_{n}\right)+\mathrm{e}_{n}
$$

where the $\mathrm{e}_{n}{ }^{\prime} s$ are zero-mean independent random variables (RVs) with variance $\sigma_{n}^{2}$, independent of $\mathcal{P}(\mathbf{x})$. The distortion due to measurement errors is defined as

$$
D \triangleq \mathbb{E}\left\{\sum_{n \in \mathcal{N}\left(\boldsymbol{\Pi}_{\mathcal{A}}\right)}\left|e_{n}\right|^{2}\right\} .
$$

Consider the estimation performed by a LSI interpolator $\theta(\mathbf{x})$ with Fourier transform $\Theta(\boldsymbol{\nu})$. In the $d$-dimensional domain, the term space-invariant takes the place of the usual time-invariant in the one-dimension domain. The estimated signal is given by

$$
\hat{\mathbf{z}}(\mathbf{x})=\left(\mathbf{z}_{\epsilon} * \theta\right)(\mathbf{x})=\sum_{n \in \mathcal{N}\left(\boldsymbol{\Pi}_{\mathcal{A}}\right)} \hat{\mathbf{z}}_{n} \theta\left(\mathbf{x}-\mathbf{x}_{n}\right) .
$$

Two metrics are employed to evaluate the signal estimation accuracy: the ESD of the estimated signal (6) and the NEMSE. The former is

$$
\mathcal{E}_{\hat{\mathbf{z}}}(\boldsymbol{\nu}) \triangleq \mathcal{F}\left\{\int_{\mathbb{R}^{d}} \mathbb{E}\left\{\hat{\mathbf{z}}(\mathbf{x}) \hat{\mathbf{z}}^{\dagger}(\mathbf{x}-\boldsymbol{\tau})\right\} d \boldsymbol{\tau}\right\}=\mathbb{E}\left\{|\hat{\mathbf{Z}}(\boldsymbol{\nu})|^{2}\right\}
$$

with $\hat{Z}(\boldsymbol{\nu}) \triangleq \mathcal{F}\{\hat{\mathbf{z}}(\mathbf{x})\}$. The latter is

$$
\epsilon_{\mathrm{S}} \triangleq \frac{\int_{\mathbb{R}^{d}}|z(\mathbf{x})-\hat{\mathbf{z}}(\mathbf{x})|^{2}}{E_{z}}
$$

where the expectation is with respect the measurement errors and the samples positions. To perform an asymptotic analysis for large sample intensity, we also introduce the following quantities normalized to the signal bandwidth-per-dimension $B_{z}:{ }^{2}$ the (normalized) spatial frequency $\breve{\nu} \triangleq \nu / 2 B_{z}$, the Poisson sampling process intensity $\breve{\rho} \triangleq \rho /\left(2 B_{z}\right)^{d}$, distortion $\breve{D} \triangleq \frac{D}{E_{z}\left(2 B_{z}\right)^{d}}$, signal ESD $\breve{\mathcal{E}}_{z}(\breve{\boldsymbol{\nu}}) \triangleq \frac{\left(2 B_{z}\right)^{d}}{E_{z}} \mathcal{E}_{z}\left(2 B_{z} \breve{\boldsymbol{\nu}}\right)$, and estimated signal ESD $\breve{\mathcal{E}}_{\hat{\mathbf{z}}}(\breve{\boldsymbol{\nu}}) \triangleq \frac{\left(2 B_{z}\right)^{d}}{E_{z}} \mathcal{E}_{\hat{\mathbf{z}}}\left(2 B_{z} \breve{\boldsymbol{\nu}}\right)$.

\section{SignAL ESTIMATION}

Lemma 1 (Optimal LSI Interpolator): The transfer function of the LSI which minimizes the NEMSE defined in (8) is given by

$$
\Theta(\boldsymbol{\nu})=\frac{\mathcal{E}_{z}(\boldsymbol{\nu})}{\rho \mathcal{E}_{z}(\boldsymbol{\nu})+E_{z}\left(1+\frac{D}{E_{z} \rho}\right)} .
$$

Proof: By extending the Wiener filtering theory to $\mathbb{R}^{d}$ as done in [15], it can be shown that the LSI interpolator minimizing $(8)$ results in

$$
\Theta(\boldsymbol{\nu})=\frac{Z(\boldsymbol{\nu}) \mathcal{U}_{\mathbf{z}_{\epsilon}}^{\dagger}(\boldsymbol{\nu})}{\mathcal{E}_{\mathbf{z}_{\epsilon}}(\boldsymbol{\nu})}
$$

where $Z(\boldsymbol{\nu}) \triangleq \mathcal{F}\{z(\mathbf{x})\}, \mathcal{U}_{\mathbf{z}_{\epsilon}}(\boldsymbol{\nu}) \triangleq \mathcal{F}\left\{\mathbb{E}\left\{\mathbf{z}_{\epsilon}(\mathbf{x})\right\}\right\}$, and $\mathcal{E}_{\mathbf{z}_{\epsilon}}(\boldsymbol{\nu}) \triangleq \mathcal{F}\left\{R_{\mathbf{z}_{\epsilon}}(\boldsymbol{\tau})\right\}$ with $R_{\mathbf{z}_{\epsilon}}(\boldsymbol{\tau}) \triangleq$

\footnotetext{
${ }^{2}$ For finite energy signals with infinite band in $\mathbb{R}^{d}$, we consider, for normalization purpose, the extension of the well-known Gabor's bandwidth to $\mathbb{R}^{d}$, i.e. $B_{z} \triangleq \sqrt{\int_{\mathbb{R}^{d}} \frac{|\boldsymbol{\nu}|^{2} \mathcal{E}_{z}(\boldsymbol{\nu}) d \nu}{E_{z}}}$.
}

$\int_{\mathbb{R}^{d}} \mathbb{E}\left\{\mathbf{z}_{\epsilon}(\mathbf{x}) \mathbf{z}_{\epsilon}^{\dagger}(\mathbf{x}-\boldsymbol{\tau})\right\} d \mathbf{x}$. In the sense of distributions, we have from (3), (4), and (6) that

$$
\mathbf{z}_{\epsilon}(\mathbf{x})=z(\mathbf{x}) \sum_{n \in \mathcal{N}(\boldsymbol{\Pi})} \delta\left(\mathbf{x}-\mathbf{x}_{n}\right)+\sum_{n \in \mathcal{N}\left(\boldsymbol{\Pi}_{\mathcal{A}}\right)} \mathrm{e}_{n} \delta\left(\mathbf{x}-\mathbf{x}_{n}\right)
$$

where the first term follows by the definition of support of $z(\mathbf{x})$. From (2a), (2b), (11), and the independence between $\mathrm{e}_{n}$ and $\mathbf{x}_{n}$, it follows that

$$
\begin{aligned}
\mathbb{E}\left\{\mathbf{z}_{\epsilon}(\mathbf{x}) \mathbf{z}_{\epsilon}^{\dagger}(\mathbf{x}-\boldsymbol{\tau})\right\}=z(\mathbf{x}) z^{\dagger}(\mathbf{x}-\boldsymbol{\tau})\left[\rho^{2}+\rho \delta(\boldsymbol{\tau})\right] \\
+\mathbb{E}\left\{\sum_{n \in \mathcal{N}\left(\boldsymbol{\Pi}_{\mathcal{A}}\right)} \sigma_{n}^{2} \delta\left(\mathbf{x}-\mathbf{x}_{n}\right) \delta\left(\mathbf{x}-\boldsymbol{\tau}-\mathbf{x}_{n}\right)\right\}
\end{aligned}
$$

and

$$
\begin{aligned}
& \mathbb{E}\left\{\mathbf{z}_{\epsilon}(\boldsymbol{\tau})\right\}=\rho z(\boldsymbol{\tau}) \\
& R_{\mathbf{z}_{\epsilon}}(\boldsymbol{\tau})=\left[\rho^{2}+\rho \delta(\boldsymbol{\tau})\right] \int_{\mathbb{R}^{d}} z(\mathbf{x}) z^{\dagger}(\mathbf{x}-\boldsymbol{\tau}) d \mathbf{x}+D \delta(\boldsymbol{\tau})
\end{aligned}
$$

By Fourier transforming (12) in $\mathbb{R}^{d}$ we obtain

$$
\begin{aligned}
& \mathcal{U}_{\mathbf{z}_{\epsilon}}(\boldsymbol{\nu})=\rho Z(\boldsymbol{\nu}) \\
& \mathcal{E}_{\mathbf{z}_{\epsilon}}(\boldsymbol{\nu})=\rho^{2} \mathcal{E}_{z}(\boldsymbol{\nu})+E_{z}\left(\rho+\frac{D}{E_{z}}\right)
\end{aligned}
$$

which, together with (10) and $\mathcal{E}_{z}(\boldsymbol{\nu})=|Z(\boldsymbol{\nu})|^{2}$, provides (9).

Remark 1: By using normalized quantities, for $\breve{\rho} \rightarrow+\infty$, the optimal LSI interpolator in (9) tends to $\frac{1}{\rho} \mathbf{1}_{\mathcal{B}_{z}}(\boldsymbol{\nu})$ where $\mathbf{1}_{\mathcal{B}_{z}}(\boldsymbol{\nu})$ denotes the indicator function equal to 1 for $\boldsymbol{\nu} \in \mathcal{B}_{z}$ and 0 otherwise. It means that the ILP filter considered in [6], [8], [9] is an asymptotic optimal choice when the samples intensity which is much higher than the signal band cardinality in $\mathbb{R}^{d}$

Remark 2: In the particular case of $D=0$ and $d=1$, (9) reduces to the optimal linear time invariant interpolator for stationary Poisson sampling process found by Leneman in [16] once the ESD is replaced by the power spectral density.

Corollary 1 (Normalized Estimated Signal ESD): When the optimal LSI interpolator in (9) is employed, the normalized ESD of the estimated signal is given by

$$
\breve{\mathcal{E}}_{\hat{\mathbf{z}}}(\breve{\boldsymbol{\nu}})=\breve{\mathcal{E}}_{z}(\breve{\boldsymbol{\nu}})\left[\frac{\breve{\mathcal{E}}_{z}(\breve{\boldsymbol{\nu}})}{\breve{\mathcal{E}}_{z}(\breve{\boldsymbol{\nu}})+\frac{1}{\check{\rho}}\left(1+\frac{\breve{D}}{\breve{\rho}}\right)}\right] .
$$

Proof: From (6) and (7), the ESD of the estimated signal $\hat{\mathrm{z}}(\boldsymbol{\nu})$ results in $\mathcal{E}_{\hat{\mathbf{z}}}(\boldsymbol{\nu})=|\Theta(\boldsymbol{\nu})|^{2} \mathcal{E}_{\mathbf{z}_{\epsilon}}(\boldsymbol{\nu})$, which from (13b) and (9) becomes (14) in terms of normalized quantities.

Remark 3: While the use of an ILP interpolator in a PPP causes a white noise floor on the estimated signal ESD [5], the optimal LSI interpolator introduces only a scaling factor point outs in square brackets in (14).

We now introduce two examples.

Example 1 (Bessel-type autocorrelation function): Consider a signal $z(\mathbf{x})\left(\mathbf{x} \in \mathbb{R}^{2}\right)$ with a Bessel-type autocorrelation function such that its normalized ESD results in $\breve{\mathcal{E}}_{z}(\breve{\boldsymbol{\nu}})=$ $\frac{4}{\pi} \mathbf{1}_{\mathcal{C}_{0}}(\breve{\boldsymbol{\nu}})$, where $\mathcal{C}_{0}$ denotes the 2 -dimensional ball centered in the origin with radius $1 / 2$. The corresponding normalized 


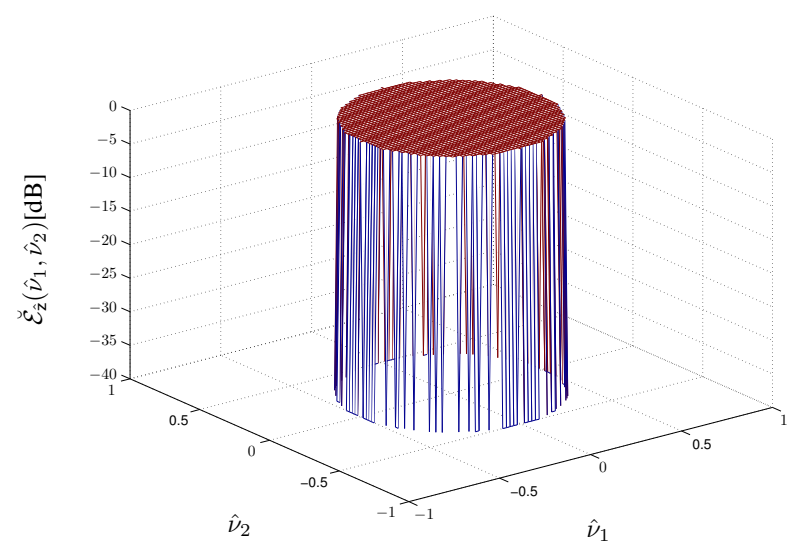

(a) The case of Bessel-type autocorrelation function

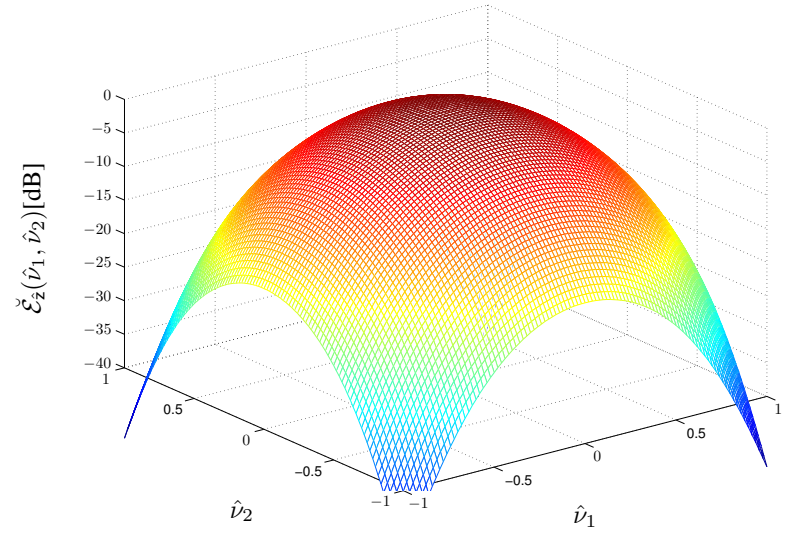

(b) The case of Gaussian autocorrelation function

Fig. 1. Estimated signal normalized ESD in $\mathbb{R}^{2}$ for $\breve{\rho}=10^{2}$ and $\breve{D}=10^{2}$.

estimated signal ESD according to (14) is depicted in Fig. 1(a).

Example 2 (Gaussian-type autocorrelation function): Consider a signal $z(\mathbf{x})\left(\mathbf{x} \in \mathbb{R}^{2}\right)$ with a Gaussian-type autocorrelation function, such that its normalized ESD results in $\breve{\mathcal{E}}_{z}(\breve{\boldsymbol{\nu}})=\frac{4}{\pi} e^{-4|\breve{\boldsymbol{\nu}}|^{2}}$ (i.e., an infinite band signal where the standard deviation is considered as the practical bandwidth per dimension for normalization purpose). The corresponding estimated signal normalized ESD according to (14) is depicted in Fig. 1(b).

Note that, in both the examples, the typical noise floor due to homogeneous Poisson sampling and measurement errors does not arise when using the optimal LSI interpolator (9).

Corollary 2 (NEMSE): When the optimal LSI interpolator in (9) is employed, the NEMSE results in

$$
\epsilon_{\mathrm{S}}=1-\int_{\mathbb{R}^{d}} \frac{\breve{\mathcal{E}}_{z}^{2}(\breve{\boldsymbol{\nu}})}{\breve{\mathcal{E}}_{z}(\breve{\boldsymbol{\nu}})+\frac{1}{\breve{\rho}}\left(1+\frac{\breve{D}}{\breve{\rho}}\right)} d \breve{\boldsymbol{\nu}}
$$

Proof: By using the fundamental isometry presented in [4], the NEMSE corresponding to the optimal LSI interpolator results in

$$
\epsilon_{\mathrm{S}}=1-\frac{1}{E_{z}} \int_{\mathbb{R}^{d}} \frac{\left|\mathcal{E}_{z, \mathbf{z}_{\epsilon}}(\boldsymbol{\nu})\right|^{2}}{\mathcal{E}_{\mathbf{z}_{\epsilon}}^{\dagger}(\boldsymbol{\nu})} d \boldsymbol{\nu}
$$

which, from (13a) and (13b), provides (15) in terms of normalized quantities.

Remark 4: From (15), the distortion effect can be described by an equivalent diminished normalized intensity

$$
\breve{\rho}_{\breve{D}} \triangleq \frac{\breve{\rho}}{1+\frac{\breve{D}}{\breve{\rho}}} .
$$

Example 3 (NEMSE for ILP ESD Signals): For signals of the example 1 , the NEMSE results in

$$
\epsilon_{\mathrm{S}}=\left(\frac{1}{\breve{\rho}_{\breve{D}}}\right) /\left(1+\frac{1}{\breve{\rho}_{\breve{D}}}\right)
$$

TABLE I

TABLE OF THE MAIN QUANTITIES RELATED TO AN LARGE WSN FOR SIGNAL ESTIMATION ( $u$ IS THE MEASUREMENT UNIT FOR THE SIGNAL).

\begin{tabular}{|lll|}
\hline Quantity & Significance & Unit \\
\hline$z(\mathbf{x})$ & Signal to be reconstructed & $\mathrm{u}$ \\
$\mathbf{x}$ & Spatial coordinate in $\mathbb{R}^{d}$ & $\mathrm{~m}$ \\
$\mathbf{x}_{n}$ & Random position of the $n$-th sample in $\mathbb{R}^{d}$ & $\mathrm{~m}$ \\
$\hat{\mathbf{z}}_{n}$ & $n$-th random sample of $z(\mathbf{x}) \in \mathbb{C}(4)$ & $\mathrm{u}$ \\
$\boldsymbol{\nu}$ & Spatial frequency coordinate in $\mathbb{R}^{d}$ & $\mathrm{~m}^{-1}$ \\
$E_{z}$ & Energy of the signal & $\mathrm{u}^{2} \mathrm{~m}^{d}$ \\
$\mathcal{E}_{z}(\boldsymbol{\nu})$ & ESD of $z(\mathbf{x})$ & $\mathrm{u}^{2} \mathrm{~m}^{2 d}$ \\
$|\mathcal{A}|$ & Cardinality of $z(\mathbf{x})$ domain in $\mathbb{R}^{d}$ & $\mathrm{~m}^{d}$ \\
$B_{z}$ & Bandwidth-per-dimension of $z(\mathbf{x})$ & $\mathrm{m}^{-1}$ \\
$D$ & Distortion due to sensing errors & $\mathrm{u}^{2}$ \\
\hline$E_{\mathrm{M}}$ & Overall estimation energy & $\mathrm{J}$ \\
$T$ & Time duration of the sensing & $\mathrm{s}$ \\
$\sigma_{\mathrm{M}}^{2}$ & Measurement error variance for each sensor & $\mathrm{u}^{2}$ \\
$W_{\mathrm{s}}$ & Power consumption for each sensor & $\mathrm{W}$ \\
$\kappa_{\mathrm{M}}$ & Proportional constant between $W_{\mathrm{s}} T$ and $1 / \sigma_{\mathrm{M}}^{2}$ & $\mathrm{~J} \mathrm{u}^{2}$ \\
$c$ & Capacity-per-volume & $\frac{\text { symbol/ch. use }}{\mathrm{m}}$ \\
\hline$\cdot$ & Normalized version of a quantity & \\
\hline
\end{tabular}

Example 4 (NEMSE for Gaussian ESD Signals): For signals of the example 2, the NEMSE results in

$$
\epsilon_{\mathrm{S}}=\frac{\pi}{4 \breve{\rho}_{\breve{D}}} \ln \left(1+\frac{4 \breve{\rho}_{\breve{D}}}{\pi}\right)
$$

Fig. 2 shows the NEMSE as a function of the normalized sampling intensity $\breve{\rho}$ for different values of the normalized distortion for both examples 3 and 4 . It can be noticed that the distortion effects vanish for $\breve{\rho}$ approaching the infinity. The different asymptote in the case of Gaussian ESD is due to its infinite signal bandwidth.

\section{Estimation ENERGy CONSTRAinT}

Consider the energy spent by the $n$-th sensor for the estimation of its own $1 / \sigma_{n}^{2}$ trough the hardware-dependent constant $\kappa_{\mathrm{M}}$ expressed in [ $\mathrm{J} \mathrm{u}^{2}$ ] where $\mathrm{u}$ is the measurement unit for the signal (see Table IV). For $\sigma_{n}^{2}=\sigma_{\mathrm{M}}^{2}$ for all $n$, the overall estimation energy is $E_{\mathrm{M}}=\kappa_{\mathrm{M}} \frac{\rho|\mathcal{A}|}{\sigma_{\mathrm{M}}^{2}}$ and the distortion 


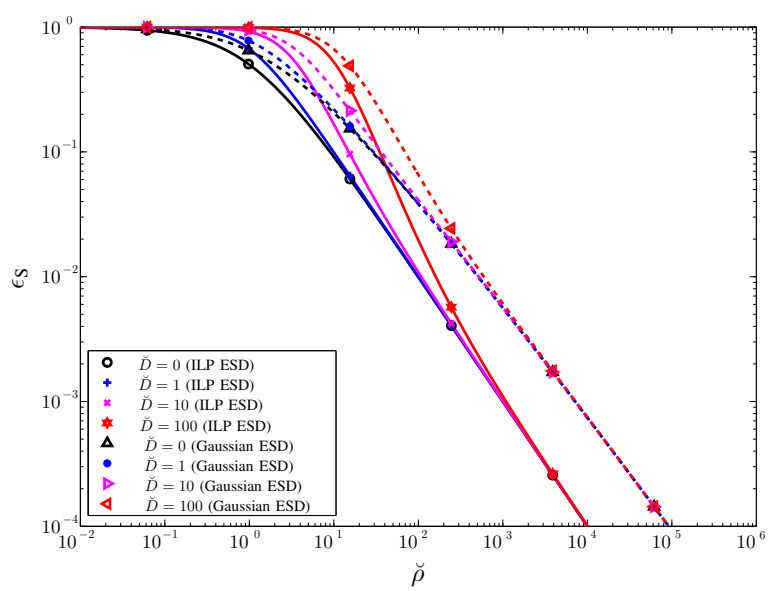

Fig. 2. NEMSE as a function of normalized sampling intensity

results in $D=\rho|\mathcal{A}| \sigma_{\mathrm{M}}^{2}$. It follows that $\breve{D}=\kappa_{\mathrm{M}} \breve{\rho}^{2} \frac{|\mathcal{A}|^{2}\left(2 B_{z}\right)^{d}}{E_{\mathrm{M}} E_{z}}$ which, substituted in (15), provides

$$
\epsilon_{\mathrm{S}}=1-\int_{\mathbb{R}^{d}} \frac{\breve{\mathcal{E}}_{z}^{2}(\breve{\boldsymbol{\nu}})}{\breve{\mathcal{E}}_{z}(\breve{\boldsymbol{\nu}})+\frac{1}{\breve{\rho}}+\kappa_{\mathrm{M}} \frac{|\mathcal{A}|^{2}\left(2 B_{z}\right)^{d}}{E_{\mathrm{M}} E_{z}}} d \breve{\boldsymbol{\nu}} .
$$

Equation (17) shows that the NEMSE is decreasing with respect to the energy spent for the estimation $E_{\mathrm{M}}$ and increasing with respect to the signal domain Lebesgue measure $|\mathcal{A}|$ and to the signal band Lebesgue measure $\left(2 B_{z}\right)^{d}$. The fact that (17) is also decreasing with respect the signal energy $E_{z}$ is simply due to the normalization choice in (8).

Remark 5: Given the overall estimation energy $E_{\mathrm{M}}$, (17) shows that having more samples (higher $\breve{\rho}$ ) with higher measurement error variance $\sigma_{\mathrm{M}}^{2}$ reduces the NEMSE with respect to the case of less samples with higher precision. This is thank to the employment of the optimal LSI interpolator (9).

Remark 6: For every finite set of samples, the NEMSE is lower bounded by $\epsilon_{\mathrm{S}}>1-\int_{\mathbb{R}^{d}} \frac{\overrightarrow{\mathcal{E}}_{z}^{2}(\breve{\boldsymbol{\nu}})}{\breve{\mathcal{E}}_{z}(\breve{\boldsymbol{\nu}})+\kappa_{\mathrm{M}} \frac{|\mathcal{A}|^{2}\left(2 B_{z}\right)^{d}}{E_{\mathrm{M}} E_{z}}} d \breve{\boldsymbol{\nu}}$. Note that such a bound is asymptotically approached for both the normalized samples intensity and the normalized distortion approaching the infinity.

\section{Network Capacity Constraint}

Consider now large WSN with constraints in the capacity of each sensor of sending the samples to the interpolation entity. Here we study the effect of network capacity constraint when a large WSN is employed to reconstruct the signal $z(\mathbf{x})$ (expressed in unit) in $\mathcal{A} \in \mathbb{R}^{2}$. Assume that the sensor positions are the output of an homogeneous PPP with intensity $\lambda$ and that each sensor is able to transmit the samples to the entity performing the interpolation with probability $q$. Thus, the sampling point process results in (1) with $\rho=q \lambda$, that is

$$
\breve{\rho}=\frac{q \lambda}{\left(2 B_{z}\right)^{d}} .
$$

\section{A. Capacity-per-unit volume Constraint}

A general and simple way to model the network capability to collect data and forward them to the interpolation entity is to

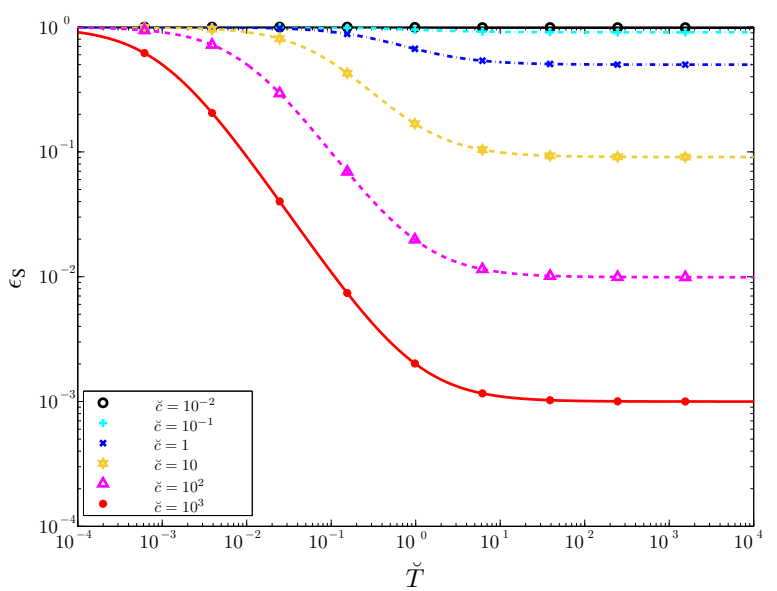

Fig. 3. Large WSN for signal estimation: MSE as a function of normalized capacity and lifetime

assume the WSN can guarantee a certain capacity-per-volume $c$. Such a value is a function of network bandwidth and protocols (MAC, routing, physical layer, etc. ${ }^{3}$ ). For homogeneous Poisson distribution of the sensors positions, the capacity of the channel between the $n$-th sensor and the interpolator for large WSN is

$$
C_{n}=\frac{C}{\left|\boldsymbol{\Pi}_{\mathcal{A}}\right|} \approx \frac{c|\mathcal{A}|}{\mathbb{E}\left\{\left|\boldsymbol{\Pi}_{\mathcal{A}}\right|\right\}}=\frac{c}{\lambda}
$$

where $C$ is the overall capacity in [symbols/channel use] and $|\cdot|$ denotes the cardinality. If the channel between the $n$-th sensor and the interpolator is modeled as an erasure channel, it results

$$
C_{n}=1-\epsilon
$$

where $\epsilon$ is the erasure probability, which is considered equal for all sensors. From (19) and (20) it follows that the sample availability can be written as

$$
q \triangleq 1-\epsilon=C_{n} \approx \frac{c}{\lambda}
$$

Since $0 \leq C \leq 1$ and $\left|\Pi_{\mathcal{A}}\right| \geq 1$ (for each PPP realization, a WSN is obviously constituted by at least one sensor), it is $0 \leq q \leq 1$.

\section{B. Sensor Lifetime Constraint}

Consider all sensors have the same power consumption $W_{\mathrm{s}}$ such that the measurement error variance results in

$$
\sigma_{\mathrm{M}}^{2}=\frac{\kappa_{\mathrm{M}}}{W_{\mathrm{s}} T}, \quad \forall n
$$

where $T$ is the estimation process duration in seconds. By substituting (22) in (5) we obtain $D=\rho|\mathcal{A}| \frac{\kappa_{\mathrm{M}}}{W_{\mathrm{s}} T}$, thus

$$
\frac{\breve{D}}{\breve{\rho}}=\frac{\kappa_{\mathrm{M}}|\mathcal{A}|}{E_{z} W_{\mathrm{s}} T}
$$

\footnotetext{
${ }^{3}$ For the impact of fading and interference see, e.g., [25] and [26].
} 


\section{NEMSE for a large WSN}

By substituting (21) in (18) we obtain

$$
\breve{\rho} \approx \frac{c}{\left(2 B_{z}\right)^{d}}
$$

that highlights how in a large WSN, the effectively available samples intensity depends on the capacity-per-volume (and no more on the sensors intensity). From (15), (23), and (24), we obtain

$$
\epsilon_{\mathrm{S}}=1-\int_{\mathbb{R}^{d}} \frac{\breve{\mathcal{E}}_{z}^{2}(\breve{\boldsymbol{\nu}})}{\breve{\mathcal{E}}_{z}(\breve{\boldsymbol{\nu}})+\frac{1}{\breve{c}}\left(1+\frac{1}{\breve{T}}\right)} d \breve{\boldsymbol{\nu}}
$$

where $\breve{c} \triangleq \frac{c}{\left(2 B_{z}\right)^{d}}$ and $\breve{T} \triangleq \frac{E_{z} W_{\mathrm{s}} T}{\kappa_{\mathrm{M}}|\mathcal{A}|}$ are two fundamental parameters for the network capacity and the sensors battery lifetime, respectively.

Remark 7: If the signal is rigorously band-limited, from (25) and for large $\breve{c}$ it is

$$
\epsilon_{\mathrm{S}}=\frac{1}{\breve{c}}\left(1+\frac{1}{\breve{T}}\right)+o\left(\frac{1}{\breve{c}}\right) .
$$

Example 5: In the case of Bessel-type autocorrelation function for the signal, it results $\epsilon_{\mathrm{S}}=\frac{1}{\breve{c}}\left(1+\frac{1}{\widetilde{T}}\right) /\left[1+\frac{1}{\breve{c}}\left(1+\frac{1}{\widetilde{T}}\right)\right]$. Fig. 3 shows the NEMSE as a function of the normalized estimation time for different values of normalized capacity. It can be seen the capacity-limited region for large $\breve{T}$, corresponding to the horizontal asymptotes, and the energy-limited region for low $\breve{T}$.

Remark 8: For infinite normalized estimation time, the asymptotical MSE is given by

$$
\epsilon_{\mathrm{S}}^{(\infty)} \triangleq \lim _{\breve{T} \rightarrow+\infty} \epsilon_{\mathrm{S}}=1-\int_{\mathbb{R}^{d}} \frac{\breve{\mathcal{E}}_{z}^{2}(\breve{\boldsymbol{\nu}})}{\breve{\mathcal{E}}_{z}(\breve{\boldsymbol{\nu}})+\frac{1}{\breve{c}}} d \breve{\boldsymbol{\nu}} .
$$

\section{CONCLUSION}

The estimation of a finite-energy signal from its samples affected by measurement errors and scattered in $\mathbb{R}^{d}$ according to an homogeneous PPP has been analyzed. The expression of the optimal LSI interpolator in the MSE sense has been derived and verified that such an expression in $\mathbb{R}^{d}$ includes a result previously known in the literature as special case.

When the optimal interpolator is used, the effect of both the random sampling and the measurement errors on the estimated signal ESD is an attenuation of the original signal ESD instead of a noise floor as for the case of ILP interpolator. Moreover, the effect of the distortion due to measurement errors on the NEMSE is shown to be equivalent to that of a reduction of samples intensity, which can be compensated by increasing the number of nodes inside the sampling area.

If a constraint in the overall estimation energy is imposed, we verified that an increasing number of sensors leads to a decreasing NEMSE in spite of the corresponding increasing measurement error for each sensor. For the case when the energy constraint is imposed on each sensor due to the battery lifetime limitation, as usual in the large WSN scenario, we derived a simple but significant expression for the NEMSE as a function of the estimation time and the capacity-per-volume.

\section{REFERENCES}

[1] R. Verdone, D. Dardari, G. Mazzini, and A. Conti, Wireless Sensor and Actuator Networks: technologies, analysis and design. Elsevier, 2008.

[2] H. Landau, "Necessary density conditions for sampling and interpolation of certain entire functions," Acta Math., vol. 117, pp. 37-52, 1967.

[3] — "Sampling data transmission, and the Nyquist rate," Proc. IEEE, vol. 55, no. 10, pp. 1701-1706, 1967.

[4] F. A. Marvasti, Non Uniform Sampling: Theory and Practice. Springer, Jun. 2001.

[5] - "Signal recovery from nonuniform samples and spectral analysis on random nonuniform samples," in Proc. IEEE Int. Conf. Acoustics, Speech, and Signal Processing, Tokyo, Japan, 1986, pp. 1649-1652.

[6] D. Dardari, A. Conti, C. Buratti, and R. Verdone, "Mathematical evaluation of environmental monitoring estimation error through energyefficient wireless sensor networks," IEEE Trans. Mobile Comput., vol. 6, no. 7, pp. 790-802, jul 2007.

[7] A. Kumar, P. Ishwar, and K. Ramchandran, "High-resolution distributed sampling of bandlimited fields with low-precision sensors," IEEE Trans. Inf. Theory, vol. 57, no. 1, pp. 476-492, Jan. 2011.

[8] J. Matamoros, F. Fabbri, C. Antón-Haro, and D. Dardari, "On the estimation of randomly sampled 2D spatial fields under bandwidth constraints," IEEE Trans. Wireless Commun., vol. 10, no. 12, pp. 1750 - 1761, Dec. 2011.

[9] F. Zabini and A. Conti, "Process estimation from randomly deployed wireless sensors with position uncertainty," in Globecom, Houston, USA, Dec. 2011.

[10] N. Sun and J. Wu, "Optimum sampling in spatial-temporally correlated wireless sensor networks," EURASIP Journal on Wireless Communications and Networking, vol. 2013, 2013, doi:10.1186/1687-1499-2013-5, 18 pages.

[11] M. Z. Win, P. C. Pinto, and L. A. Shepp, "A mathematical theory of network interference and its applications," Proc. IEEE, vol. 97, no. 2, pp. 205-230, Feb. 2009.

[12] E. Salbaroli and A. Zanella, "Interference analysis in a Poisson field of nodes of finite area," IEEE Trans. Veh. Technol., vol. 58, no. 4, pp. 1776-1783, May 2009.

[13] M. Haenggi, J. Andrews, F. Baccelli, O. Dousse, and M. Franceschetti, "Stochastic geometry and random graphs for the analysis and design of wireless networks," IEEE J. Sel. Areas Commun., vol. 27, no. 7, pp. 1029-1046, Sep. 2009.

[14] A. Rabbachin, A. Conti, and M. Z. Win, "Wireless network intrinsic secrecy," IEEE/ACM Trans. Netw., vol. 23, no. 1, pp. 56-69, Feb 2015.

[15] F. Zabini and A. Conti, "Inhomogeneous Poisson sampling of finiteenergy signals with uncertainties in $\mathbb{R}^{d}$," IEEE Trans. Signal Process., pp. 1-16, 2016, early access.

[16] O. A. Z. Leneman, "Random sampling of random processes: Optimum linear interpolation," J. of Franklin Inst., vol. 4, no. 281, pp. 302-314, 1966.

[17] — "Random sampling of random processes: Impulse processes," Inf. and Control, no. 9, pp. 347-363, 1966.

[18] O. A. Z. Leneman and J. B. Lewis, "Random sampling of random processes, mean-square comparison of various interpolators," IEEE Trans. Autom. Control, vol. AC, no. 11, pp. 396-403, 1966.

[19] A. Nordio, C. F. Chiasserini, and E. Viterbo, "Performance of linear field reconstruction techniques with noise and uncertain sensor locations," IEEE Trans. Signal Process., vol. 56, no. 8, pp. 3535,3547, Aug. 2008.

[20] B. Csáji, M. C. Campi, and E. Weyer, "Non-asymptotic confidence regions for the least-squares estimate," in Proc. IFAC SYSID, Brussels, Belgium, Jun. 2012, pp. 227-232.

[21] V. Zambianchi, M. Kieffer, F. Bassi, G. Pasolini, and D. Dardari, "Distributed sps algorithms for non-asymptotic confidence region evaluation," in Networks and Communications (EuCNC), 2014 European Conference on, Jun. 2014, pp. 1-5.

[22] E. M. Stein and G. Weiss, Introduction To Fourier Analysis on Euclidean Spaces. Princeton University Press, 1971.

[23] J. F. Kingman, Poisson Processes. Oxford University Press, 1st edition, 1993.

[24] A. Papoulis and S. U. Pillai, Probability, Random Variables and Stochastic Processes. McGraw-Hill, 2002.

[25] F. Zabini, B. Masini, A. Conti, and L. Hanzo, "Partial equalization for MC-CDMA systems in non-ideally estimated correlated fading," IEEE Trans. Veh. Technol., vol. 59, no. 8, pp. 3818-3830, Oct. 2010.

[26] A. Zanella, A. Bazzi, G. Pasolini, and B. M. Masini, "On the impact of routing strategies on the interference of ad hoc wireless networks," IEEE Trans. Commun., vol. 61, no. 10, pp. 4322-4333, October 2013. 